\title{
Analysis of Trend of Studies on Microneedle Treatment System (MTS)
}

\author{
Hea-Sun Chun, Ho-Seub Song*
}

Department of Acupuncture \& Moxibustion Medicine, College of Korean Medicine, Gachon University, Seongnam, Republic of Korea

Received October 6, 2021

Reviewed October 24, 2021

Accepted October 27, 2021

*Corresponding Author

Ho-Seub Song

Department of Acupuncture \&

Moxibustion Medicine, College of Korean Medicine, Gachon University,

1342 Seongnam-daero, Sujeong-gu,

Seongnam 13120, Republic of Korea

Tel: +82-31-750-5424

E-mail: happyi0927@naver.com
Objectives: The purpose of this study was to analyze the microneedle therapy system (MTS) and its research methods for the past 10 years in Korea.

Methods: Data on microneedle therapy system were collected using NDSL, KISS, RISS, and OASIS electronic databases from January 2010 to August 2021. "microneedle," "derma stamp," "microneedle therapy system" were used as the keywords. The present study, however, excluded data that were 1) unrelated to the microneedle therapy system, 2) from review/meta/protocol studies, and 3) from overseas studies. Data selected through the primary screening process, animal studies, case reports, and clinical data were included in the analysis. However, information data not related to the microneedle therapy system were excluded from the study.

Results: Among the MTS-related papers published from January 2010 to August 2021, 7 animal research, 2 clinical trials, and 10 case studies were published. Based on the research topics, there were 8 papers on skin improvement and skin diseases, 7 papers on hair growth and hair loss, 3 papers on stability, and 1 paper on peripheral facial paralysis. Conclusion: Most of the studies related to MTS focused on skin, hair, and stability. The effect of MTS on hair growth and skin improvement has been confirmed, and it has been proven to have significant effects on the treatment of acne, acne scars, and hair loss in clinical practice. No serious side effects were observed during the MTS treatment, and the safety assessment confirmed that it was safe for use.

Keywords: acne, hair loss, microneedle therapy system, mts, review, stability

\section{INTRODUCTION}

MTS is a device designed to create multiple microscopic channels in the skin through mechanical holes wherein a 0.25 $2.0 \mathrm{~mm}$ roller equipped with about 192-200 needles is rolled on the skin surface. It is a treatment method that uses the skin's own regenerative ability to regenerate new collagen $[1,2]$.

Initially, MTS was used as a method to increase drug delivery. However, studies have reported that it is effective in improving various scars and wrinkles just by mechanically making a hole in the skin without the application of active drug ingredients [3].

MTS is a treatment that accelerates the cell replacement cycle and induces collagen production through skin stimulation. It can be said to originate from the method called skin acupunc- ture therapy (皮膚鍼療法, PIBUCHIMYOBEOB) in Oriental Medicine [4].

As the average life expectancy increases, there is a growing desire to maintain a young and healthy life rather than simply extending the lifespan [4]. Therefore, active research is also being conducted using MTS for the treatment of skin aging and hair loss. Therefore, we conducted this review to provide a basis for future MTS treatment and research by examining research trends using MTS published in Korea from January 2010 to August 2021. 


\section{MATERIALS AND METHODS}

\section{Data sources and searches}

Based on the electronic database for documents published from January 2010 to August 2021, this study was conducted using search terms such as "microneedle," "derma stamp," "microneedle therapy system" in NDSL, KISS, RISS, and OASIS.

\section{Data selection (Fig. 1)}

By retrieving the data titles in the database from January 2010 to August 2021, the study was conducted on the original text with topics containing 1) microneedle, 2) microneedle therapy system, and 3) literature in domestic academic journals and the domestic dissertations. If the original text is selected, 1) original text unrelated to the microneedle therapy system, 2) original text related to clinical review/meta/protocol studies, 3) overseas studies, and 4) original texts that do not fall under 2010-2021 were excluded from the study. The following data were included through the primary screening process: 1) animal studies, 2) case reports, and 3) clinical data. From the above, 1) no informational data and 2) data unrelated to the microneedle therapy system were excluded from the study.

\section{Data analysis}

The frequency and distribution of each item are summarized in a table. If necessary, the overall distribution was plotted in a graph, and tables and graphs were prepared using Excel 2019
(Microsoft Corp., Redmond, WA, USA).

\section{RESULTS}

The number of papers analyzed in this study is as follows (Fig. 2).

According to the type of paper, there were 7 animal research, 2 clinical trials, and 10 case studies (Fig. 3).

\section{Animal research}

The list of animal research for MTS is as follows (Table 1).

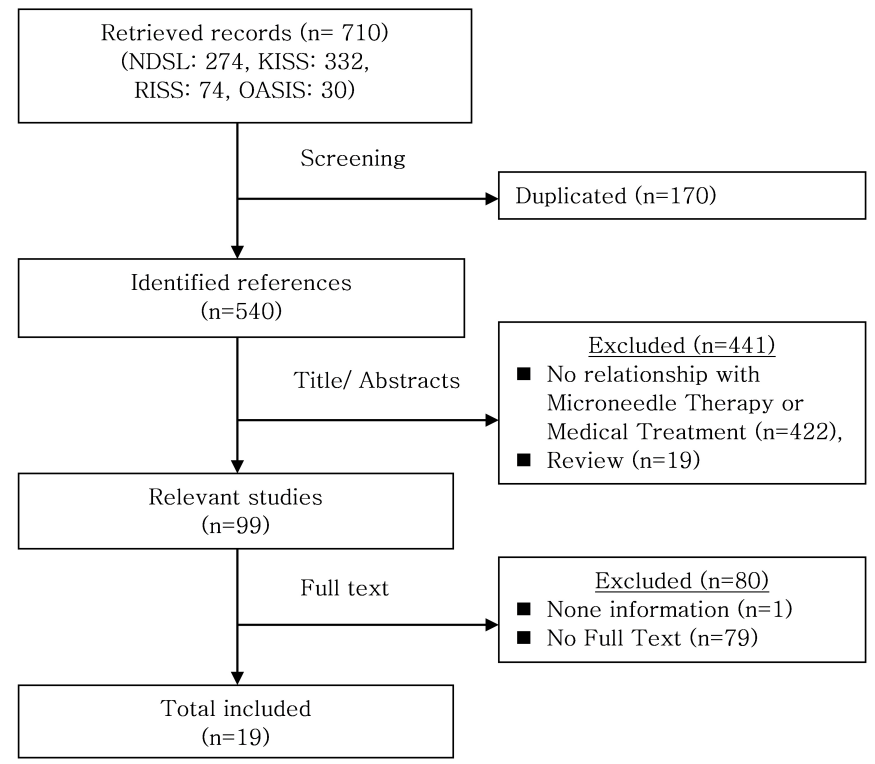

Figure 1. Reference flow chart.

2.5

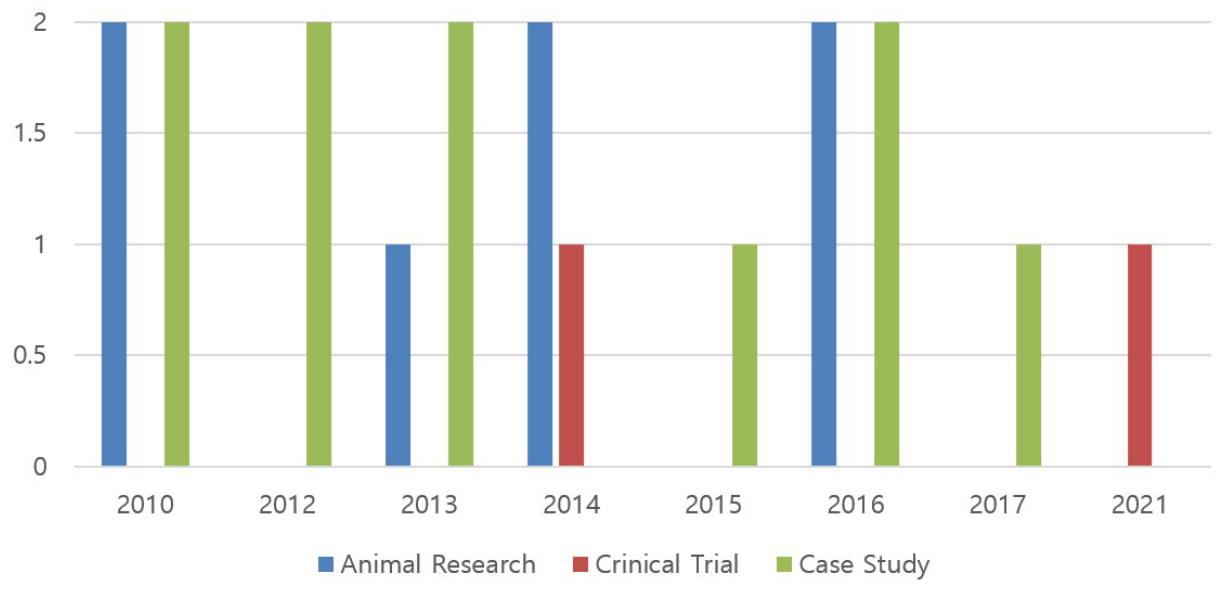

Figure 2. The number of papers by year. 


\section{1) Research animal}

Three papers were studied using hairless mice, two were studied using C57BL/6 mice, and two were studied using C57BL/6N mice.

\section{2) Research purpose}

Of the seven papers, one paper researched the dermal proliferative effect and stability of MTS, two papers researched the stability of MTS, three papers researched the hair growth effect of MTS, and one paper researched the most optimal length and the cycle of MTS for hair growth.

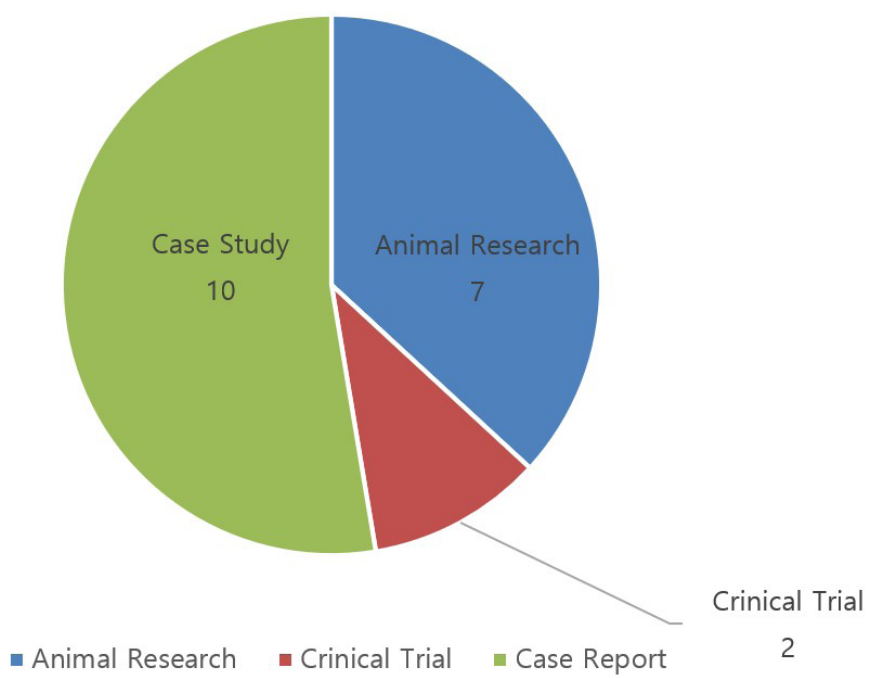

Figure 3. Classifications of paper type.

\section{3) Research method}

Kim et al. [5] evaluated the dermal proliferative effect and stability of AMTS and AMTS-H by comparing MTS and AMTS and AMTS-H. Park et al. [6] evaluated the stability of Disk type-Microneedle Therapy System (DTS) using the conventional MTS and DTS. Park et al. [7] evaluated the stability of Digital Hand ${ }^{\circledR}$ and Digital Pro ${ }^{\circledR}$ by comparing DTS with automatized stamp type microneedles Digital Hand ${ }^{\circledR}$ and Digital Pro ${ }^{\circledR}$. Lee et al. [8] studied the hair growth effect by comparing the control group, the Minoxidil group, and the MTS group. Ju et al. [9] studied the hair growth effect by comparing the control group, the Minoxidil group, the MTS group, and the MTS + Ganoderma lucidum extract (GLE) group. Kang et al. [10] studied the hair growth effect by dividing the group into four groups as follows: the control group, the Minoxidil group, the MTS group, and the MTS + Hwangryeonhaedoktang Pharmacopuncture Solution (HRHDT) group. Kim et al. [11] studied the hair growth effect according to the length and cycle of DTS.

\section{4) Assessment methods}

Kim et al. [5] evaluated efficacy after the biopsy using H\&M staining, Masson trichrome staining, and Western blot analysis. Herein, the erythema was observed by visual inspection, and the transepidermal water loss (TEWL) and stratum corneum (SC) hydration were measured using the Tewameter, whereas the stability was evaluated by observing epidermal micropore recovery and checking the number and type of inflammatory cells by biopsy. Park et al. [6] evaluated the stability by observing neutrophils, lymphocytes, and basophils using staining with

Table 1. List of animal researches for microneedle therapy system

\begin{tabular}{|c|c|c|c|c|}
\hline No. & Author & Study name & Journal & Year \\
\hline 1 & Kim JH et al. [5] & Dermal Proliferative Effect and Safety of Automicroneedle Therapy System & Korean Journal of Dermatology & 2010 \\
\hline 2 & Park KY et al. [6] & The Stability of the Disk-type Microneedle in Skh-hairless-1 (Albino) Mice & $\begin{array}{l}\text { Journal of Asthma, Allergy and } \\
\text { Clinical Immunology }\end{array}$ & 2010 \\
\hline 3 & Park KY et al. [7] & Safety Evaluation of Stamp Type Digital Microneedle Device in Hairless Mice & Ann Dermatol & 2013 \\
\hline 4 & Lee $\mathrm{CH}$ et al. [8] & Hair-growth Promoting Effect of Microneedle Roller Therapy & $\begin{array}{l}\text { Korean J. Oriental Physiology \& } \\
\text { Pathology }\end{array}$ & 2014 \\
\hline 5 & Ju BH et al. [9] & $\begin{array}{l}\text { Effects of Ganoderma Lucidum Extract Ethanol Extract and Microneedle } \\
\text { Therapy System on Hair Growth in an Alopecia Model of C57BL/6N Mice }\end{array}$ & J Pediatr Korean Med & 2014 \\
\hline 6 & Kang NR et al. [10] & $\begin{array}{l}\text { Effects of Microneedle Therapy System (MTS) and Hwangryeonhaedoktang } \\
\text { Pharmacopuncture Solution on Hair Growth in an Alopecia Model of } \\
\text { C57BL/6N Mouse }\end{array}$ & $\begin{array}{l}\text { J Korean Med Ophthalmol } \\
\text { Otolaryngol Dermatol }\end{array}$ & 2016 \\
\hline 7 & Kim YS et al. [11] & $\begin{array}{l}\text { Repeated Microneedle Stimulation Induced Enhanced Hair Growth in a } \\
\text { Murine Model }\end{array}$ & Ann Dermatol & 2016 \\
\hline
\end{tabular}


hematoxylin and eosin after the biopsy and observing erythema by visual inspection. Park et al. [7] evaluated the stability by visually observing erythema and needle marks; by observing TEWL using a Tewameter; and by observing inflammatory cell infiltration, desquamation of the stratum corneum, or disruption of the basal layer with biopsy. Lee et al. [8] analyzed hair growth effect by visual observation using dermoscopy; by observation of hair follicles with hematoxylin and eosin staining after biopsy; and by testing antibody of bromodeoxyuridine (BrdU), fibroblast growth factor (FGF), and vascular endothelial growth factor (VEGF) by immunohistochemistry and PTPCR. Ju et al. [9] and Kang et al. [10] evaluated the hair growth effect by visual observation after pulling out hair to observe the hair length. The density and diameter of the hair were observed using a Folliscope. After the biopsy, Western blot analysis was performed to confirm IGF-1 and VEGF. Hematoxylin and eosin staining was used to observe the size and number of hair follicles and the length of the hair root. Moreover, the RT-PCR was performed. In addition, the body weight and organ weight of mice were measured to check side effects and stress. Kim et al. [11] observed hair growth and hair density using photographs and a Folliscope and evaluated the hair growth effect by performing RT-PCR and immunohistochemistry with biopsy samples.

\section{Clinical trials}

The list of clinical trials for MTS is as follows (Table 2).

\section{1) Research subject}

Lee et al. [12] studied 25 women, whereas Koo [13] studied 50 ordinary people, but among them, only 38 people were analyzed due to various reasons such as dropping out of the study.

\section{2) Research and evaluation methods}

Lee et al. [12] conducted a randomized, controlled, blinded split-face study. DTS treatment, namely DTS + Human Embryonic Stem Cells Conditioned Medium (hESC-EPC CM) 0.5
$\mathrm{mL}$ treatment, was performed on both left and right sides of the face. After repeated five treatment sessions at 2-week intervals, photographs were taken and self-assessment questionnaires were conducted. In addition, color changes were evaluated using a Mexameter, whereas collagen regeneration was evaluated using a Visiometer SV600. Koo [13] performed the study into two groups: the skin toner + MTS group and the ginseng extract dissolved toner + MTS group. Then, after performing MTS treatment once a week for a total of three times, T zone oil change, pore change, a skin tone change, and pigmentation change were evaluated using an A-ONE Smart One-click automatic facial diagnostic device.

\section{Case studies}

The list of case studies for MTS is represented in Table 3.

\section{1) Research disease and patient}

Out of a total of 10 case studies, one was for patients with acne, one for patients with aging skin, four for patients with acne scars, one for patients with peripheral facial paralysis, one for a pediatric patient with different types of alopecia, and two for patients with androgenetic alopecia (AGA).

\section{2) Treatment and evaluation methods}

Seong et al. [14] studied a total of 7 cases wherein they treated acne only with the Auto-Microneedle Therapy System (AMTS) without using any herbal medicine. A total of 7 treatments were performed once a week for 7 weeks. Changes in pores, wrinkles, pigmentation, sebum, porphyrin, and skin tone were evaluated with Janus facial diagnostics. The Korean Acne Grading System (KAGS) was classified and evaluated, and digital infrared thermographic imaging (DITI) was conducted. In addition, quality of life in various skin conditions was evaluated with Skindex-29, and satisfaction was evaluated using a questionnaire.

Seong et al. [4] conducted a retrospective study analyzing charts of patients treated from July 2009 to March 2010. Derma

Table 2. List of clinical trials for microneedle therapy system

\begin{tabular}{ccccc}
\hline No. & Author & & Study name & Journal \\
\hline 1 & Lee HJ et al. [12] & $\begin{array}{c}\text { Efficacy of Microneedling Plus Human Stem Cell Conditioned Medium For Skin } \\
\text { Rejuvenation: A Randomized, Controlled, Blinded Split-Face Study }\end{array}$ & Ann Dermatol & 2014 \\
2 & Koo JS [13] & $\begin{array}{l}\text { A Study on the Skin Improvement Effect of Ginseng Extract with Microneedle Therapy } \\
\text { System }\end{array}$ & Kor. J. Herbol & 2021 \\
\hline
\end{tabular}


Table 3. List of case studies for microneedle therapy system

\begin{tabular}{|c|c|c|c|c|}
\hline No. & Author & Study name & Journal & Year \\
\hline 1 & Seong EJ et al. [14] & A Clinical report on the acne treatment with AMTS & $\begin{array}{l}\text { J Korean Med Ophthalmol } \\
\text { Otolaryngol Dermatol }\end{array}$ & 2010 \\
\hline 2 & Seong EJ et al. [4] & A Clinical report on the rejuvenate treatment with derma stamp & $\begin{array}{l}\text { J Korean Med Ophthalmol } \\
\text { Otolaryngol Dermatol }\end{array}$ & 2010 \\
\hline 3 & Lee KS et al. [15] & $\begin{array}{l}\text { Case Study of Treating Acne Scar Using Scar Regeneration Acupuncture } \\
\text { Therapy and Micro-needle Therapy }\end{array}$ & J Korean Acupunct Moxib Soc & 2012 \\
\hline 4 & Lee UI et al. [16] & $\begin{array}{l}\text { Clinical Observation of Effect on Severity of Symptoms and Safety of Auto- } \\
\text { microneedle Therapy in Patient with Peripheral Facial Paralysis }\end{array}$ & J Korean Acupunct Moxib Soc & 2012 \\
\hline 5 & Ju BH et al. [17] & A Case Report on Child with Different Types of Alopecia & J Pediatr Korean Med & 2013 \\
\hline 6 & Heo JE et al. [18] & $\begin{array}{l}\text { Case Study of Treating Acne Scars Using Reconstruction of Skin Scars and } \\
\text { Auto Microneedle Therapy System }\end{array}$ & $\begin{array}{l}\text { J Korean Med Ophthalmol } \\
\text { Otolaryngol Dermatol }\end{array}$ & 2013 \\
\hline 7 & Kim HY et al. [19] & $\begin{array}{l}\text { Clinical Stydy of Twenty-seven Case of Treating Acne scar Using } \\
\text { Microneedle Therapy and Subcision Combined with BeevenoSm } \\
\text { Pharmacopuncture Therapy }\end{array}$ & $\begin{array}{l}\text { J Korean Med Ophthalmol } \\
\text { Otolaryngol Dermatol }\end{array}$ & 2015 \\
\hline 8 & Lee HK et al. [20] & Comparative Study on Treatment of Acne Scar & $\begin{array}{l}\text { J Korean Med Ophthalmol } \\
\text { Otolaryngol Dermatol }\end{array}$ & 2016 \\
\hline 9 & Ro Bl et al. [21] & $\begin{array}{l}\text { Therapeutic Effects of Growth Factor Cocktail Treatment in Patients with } \\
\text { Androgenetic Alopecia According to the Depth of Microneedle }\end{array}$ & $\begin{array}{l}\text { Korean Journal of } \\
\text { Dermatology }\end{array}$ & 2016 \\
\hline 10 & Ro Bl et al. [22] & $\begin{array}{l}\text { Therapeutic Effects of Growth Factor Cocktail Including Fibroblast Growth } \\
\text { Factor } 9 \text { in Patients with Pattern Hair Loss }\end{array}$ & $\begin{array}{l}\text { Korean Journal of } \\
\text { Dermatology }\end{array}$ & 2017 \\
\hline
\end{tabular}

Stamp treatment was performed once a week for a total of five times. A total of 10 patients were analyzed. Among them, 2 cases received herbal medicines for symptoms such as general pain, coldness, and obesity, whereas the remaining 8 cases did not receive herbal medicines. For evaluation, a Janus facial tester was used to assess the changes in pores, wrinkles, pigmentation, sebum, porphyrin, and skin tone. In addition, satisfaction was evaluated using a questionnaire.

Lee et al. [15] treated acne scars with a Derma Microneedle Roller, a type of MTS, and scar regeneration acupuncture method. The evaluation was conducted using photographs, KAGS, Qualitative Global Acne Scarring Grading system (QGASC), and Cardiff Acne Disability Index in Korean (KCADI), whereas the satisfaction was evaluated using a questionnaire.

Lee et al. [16] studied patients with peripheral facial paralysis who were treated with AMTS two or more times on the affected face. In addition, acupuncture, pharmacopuncture, herbal medicine, electroacupuncture, intradermal acupuncture, and taping therapy were performed. Evaluation was conducted using the Yanagihara unweighted grading system, Sunnybrook Facial Grading System (SBGS), and Facial Disability Index (FDI). After treatment, stability evaluations such as needle pain and fatigue were also performed.
Ju et al. [17] performed MTS treatment on pediatric patients with complex pediatric alopecia in the frontal and temporal regions. In addition to MTS treatment, herbal medicine, acupuncture treatment on the lesion site, pharmacopuncture, scalp care, high-frequency treatment, low-frequency treatment, and cupping were performed. The treatment effect was observed using photographs.

Heo et al. [18] treated six patients with acne scars using AMTS and scar reconstruction therapy but did not use other drugs or treatments along with it. Efficacy was evaluated based on photographs and QGASC. A patient satisfaction survey was also conducted.

Kim et al. [19] studied 27 patients with acne scars who had previously visited the hospital and completed 12 procedures for the treatment of acne scars. The treatment with AMTS, Subcision + Beevenom Pharmacopuncture, AMTS, and Beevenom Pharmacopuncture was followed in the given order as one set, and a total of three sets were repeated for 12 weeks. No other medications or treatments that would affect acne were administered during the treatment period. Evaluation was conducted using KAGS, Echelle d'evaluation Clinique des Cicatrices d'Acne (ECCA), and patient satisfaction survey, and side effects were observed. 
Lee et al. [20] studied patients with acne scars from March 2015 to September 2015 and treated them for a period of 5-10 months using AMTS, Subcision, and Hwangryeonhaedoktang Pharmacopuncture. The total number of treatments ranged from less than 5 to more than 10 . According to the treatment method, 25 people were assigned to the MTS group, 27 people to the Subcision group, 20 people to the MTS + Subcision group, and 23 people to the Subcision + pharmacopuncture group. The evaluation was conducted using KAGS and QGASC, and side effects and adverse reactions were observed.

Ro et al. [21] analyzed the therapeutic effect based on the depth of microneedle in patients with AGA. The MTS depth was $0.5 \mathrm{~mm}$ and $0.3 \mathrm{~mm}$ on the right side and the left side of the scalp, respectively. Each patient received six treatments at 2 -week intervals for a period of 3 months. For the evaluation, hair density and hair diameter were observed with a phototrichogram, and photographs were used for the analysis.

Ro et al. [22] studied the effects of GFC including fibroblast growth factor 9 (FGF9) on patients with AGA. GFC with FGF9 + MTS treatment was performed on the right side of the scalp, whereas MTS + Normal saline treatment was performed on the left side of the scalp. Each patient received six treatments at 2-week intervals for a period of 3 months. In the evaluation, hair density and hair diameter were observed with a phototrichogram, and photographs were used for the analysis.

\section{DISCUSSION}

MTS is a modern skin acupuncture therapy (皮膚鍼療法, PIBUCHIMYOBEOB) developed from traditional Oriental Medicine [18].

MTS is a treatment method that regenerates the skin by making numerous microholes in the skin and using a natural wound healing mechanism along with the medicinal effects of the transdermal drug delivery system (TDDS) [23, 24].

Studies have been reported that MTS is effective in improving various scars and wrinkles just by making a microhole mechanically [3], which helps the drug to penetrate the skin more effectively by creating an effective TDDS [23].

Among various drug delivery systems (DDS), the main advantages of TDDS through the skin are that it has less toxicity to the human body, does not cause gastrointestinal disturbances, and does not have any primary metabolic process in the liver (first-pass effect) compared to oral or vascular DDS. However, despite these advantages, TDDS has several limitations to over- come. First, there is a limit to the concentration of drugs that can be delivered through the skin. Most of the drugs used in TDDS should be fat soluble and of low molecular weight [25].

Recently, various methods have been researched to overcome the limitations of TDDS. Among them, MTS is used in TDDS for the treatment of hair loss, hyperhidrosis (using botulinum toxin), and melasma (using vitamins) [6].

Accordingly, we conducted the present review to help future treatment and research using MTS by looking at research trends using MTS that have been published in Korea from January 2010 to August 2021.

According to the type of study, we analyzed 7 animal research, 2 clinical trials, and 10 case studies.

According to the research topics, there were 8 papers on skin improvement and skin diseases, 7 papers on hair growth and hair loss, 3 papers on stability, and 1 paper on peripheral facial paralysis.

In the animal research, in an experiment, hairless mice were used to determine the dermal proliferative effect or stability and C57BL/6 and C57BL/6N mice were used to determine the hair growth effect.

Kim et al. [5] carried out the study into four groups: the control group, the MTS group, the AMTS group, and the AMTS$\mathrm{H}$ group and evaluated the dermal proliferative effect and stability of AMTS and AMTS-H with biopsy, visual inspection, and Tewameter. In the AMTS and AMTS-H groups, a significant increase in dermal thickness and dermal density and an increase in procollagen-1 were confirmed. In addition, it was confirmed that all micropores of the epidermis were closed 1 $\mathrm{h}$ after the treatment and that no stability problems related to treatment, such as erythema, were found. Park et al. [6] evaluated the stability of DTS by carrying out a biopsy and visual inspection by dividing it into three groups such as control group, MTS group, and DTS group. In all groups, no significant skin inflammatory reactions or other abnormal signs other than mild erythema were observed; no significant infiltration of neutrophils, lymphocytes, or basophils was observed; and no exfoliation of the stratum corneum or destruction of the epidermis was observed. Park et al. [7] performed the study in four groups as follows: the control group, the Digital Hand ${ }^{\circledR}$ group, the Digital Pro ${ }^{\circledR}$ group, and the DTS group to observe the stability based on visual inspection, Tewameter, and biopsy. Stability was confirmed due to the absence of inflammatory cell infiltration, desquamation of the stratum corneum, or disruption of the basal layer and the absence of erythema and needle marks 
on the face on the next day after the procedure. Because Digital Hand $^{\circledR}$ and Digital Pro ${ }^{\circledR}$ are stamp methods, they have the advantages of having accurate power, are more comfortable due to their ease of insertion into the skin, and are judged to avoid the minor abrasion often caused by microneedle rollers. Lee et al. [8] divided the group into three groups: the control group, the Minoxidil group, and the MTS group. They used dermoscopy, biopsy, immunohistochemistry, and RT-PCR to examine the hair growth effect, wherein they confirmed an increase in hair density, hair diameter, and hair growth. Ju et al. [9] performed the study in four groups as follows: the control group, the Minoxidil group, the MTS group, and the MTS + GLE group and analyzed the hair growth effect by visual inspection, Folliscope, biopsy, and RT-PCR. Nonetheless, the stress and side effects were observed by measuring the body weight and organ weight of experimental mice. The promotion of hair growth such as improvement in the hair density, thickness, and length was observed in the Minoxidil group, the MTS group, and the GLE + MTS group, wherein no stress or side effects were observed. The GLE + MTS group was found to be more effective in hair growth than the MTS group. Kang et al. [10] divided the group into four groups: the control group, the Minoxidil group, the MTS group, and the MTS + HRHDT group. They analyzed the hair growth effect using visual inspection, Folliscope, biopsy, and RT-PCR and measured the weight and organ weight of mice to determine stress and side effects. The hair growth effect was significantly increased in the Minoxidil group, the MTS group, and the MTS + HRHDT group, wherein no stress or side effects were observed. The MTS + HRHDT group was found to be more effective for hair growth than the MTS group. Kim et al. [11] observed the optimal length and cycle of DTS on hair growth by observing the length and cycle of optical DTS showing the hair growth effect with photographs, Folliscope, RT-PCR, and immunohistochemistry by varying the length and cycle of DTS. It was confirmed that the optimal length and cycle of DTS on hair growth was $0.25 \mathrm{~mm} / 10$ cycles and 0.5 $\mathrm{mm} / 10$ cycles.

In two clinical trials by Lee et al. [12], randomized, controlled, blinded split-face studies, skin improvement was mainly studied.

Lee et al. [12] performed DTS treatment and DTS + hESCEPC CM $0.5 \mathrm{~mL}$ treatment on the left and right sides of the face, respectively. Using photographs, a self-assessment questionnaire, Mexameter, and Visiometer, a significant improvement in pigmentation and wrinkles was confirmed in the DTS
+ hESC-EPC CM group than that in the DTS group. Koo [13] carried out a study in the skin toner + MTS group and the ginseng extract dissolved toner + MTS group and confirmed a significant improvement in $\mathrm{T}$ zone oil reduction, pore reduction, and pigmentation in the ginseng extract dissolved toner + MTS group using the ONE Smart One-click automatic facial diagnostic device.

There were a total of 10 case studies, 4 of which were the most studied cases for patients with acne scars, and 2 other cases were the next most common for patients with AGA. There was one case report each for patients with acne, aging skin, peripheral facial paralysis, and pediatric complex type hair loss.

Seong et al. [14] confirmed the improvement in pore size, wrinkles, spots, sebum, porphyrin, and skin tone using the Janus facial diagnostic machine after treating acne using ATMS in 7 patients who visited the hospital for acne. The improvement of acne was confirmed based on KAGS and Skindex-29 outcomes. Seong et al. [4] is a retrospective chart study of 10 patients who visited the hospital for aging skin. After treatment for aging skin using Derma Stamp, improvement in pore size, wrinkles, spots, sebum, porphyrin, and skin tone was confirmed using Janus facial diagnostics. Although the treatment effect of the oily skin was superior to that of the dry skin in all aspects, treatment satisfaction was confirmed to be higher in dry skin patients. However, in this study, the average re-evaluation period was 129.8 days and the difference between the last treatment date and the last facial examination was 7-362 days, which was irregular. Lee et al. [15] treated acne scars with the Derma Microneedle Roller and scar regeneration acupuncture method for five patients with acne scars and confirmed the improvement in acne scars using photographs, KAGS, QGASC, and KCADI. Lee et al. [16] studied 27 patients with peripheral facial paralysis and treated the affected face using an MTS. In addition, acupuncture, pharmacopuncture, herbal medicine, electroacupuncture, intradermal acupuncture, and taping therapy related to peripheral facial paralysis were performed. The stability and improvement in the Yanagihara unweighted grading system, SBGS, and FDI were confirmed. It was noted that the AMTS combination treatment in peripheral facial paralysis could lead to clinical improvement, wherein the absence of a control group was considered as the limitation of the study. Ju et al. [17] performed MTS treatment along with herbal medicine treatment, lesion site acupuncture treatment, herbal acupuncture treatment, scalp care, etc. for pediatric patients with complex pediatric hair loss in the frontal and temporal regions. The 
improvement of the lesion site was confirmed by photographs. Heo et al. [18] confirmed the improvement in acne scars based on photographs and QGASC by treating six patients with acne scars using AMTS and scar reconstruction. Kim et al. [19] targeted 27 patients who visited the hospital with acne scars and had completed 12 treatments. After 12 weeks of treatment by repeating a total of 3 sets in order, significant improvement in acne scars was confirmed based on KAGS and ECCA. Lee et al. [20] divided patients with acne scars into 25 people in the MTS group, 27 people in the subcision group, 20 people in the MTS + Subcision group, and 23 people in the Subcision + pharmacopuncture group and evaluated the treatment effect according to the treatment method for acne scars using KAGS and QGASC as a standard, and improvement in acne scars was confirmed in all groups, wherein the treatment period was significantly shorter in the MTS + Subcision group. Ro et al. [21] divided the 11 cases of patients with AGA into groups treated with MTS to the depth of $0.5 \mathrm{~mm}$ and $0.3 \mathrm{~mm}$ on the right side and on the left side of the scalp, respectively. They checked the hair density and hair diameter using a phototrichogram. A significant improvement in hair density and hair diameter was confirmed in both groups. It was also confirmed that the group treated with MTS to a depth of $0.5 \mathrm{~mm}$ showed a more significant effect on hair density than the group treated with MTS to a depth of 0.3 mm. Ro et al. [22] performed MTS + GFC with FGF9 treatment and MTS + Normal saline treatment on the right side and on the left side of the scalp of 22 patients with AGA, respectively, and checked their hair density and hair diameter with a phototrichogram. Significant improvement in the hair density and hair diameter was confirmed in both the MTS + GFC with FGF9 treatment group and the MTS + Normal saline treatment group.

As such, most of the studies using MTS, including the safety evaluation, focused on skin diseases such as acne scars, acne, aging skin, and hair loss. So, the scope of the studies using MTS was limited. In addition, in most case studies, MTS was combined with other treatments. However, a limitation was that the number of clinical trials was small. Therefore, we think that various types of research are needed along with the active use of MTS in various fields, such as in skin diseases and hair loss.

\section{CONCLUSION}

1. As a result of analyzing the studies using MTS according to the study type, there were 7 animal research, 2 clinical trials, and 10 case studies.

2. As a result of analyzing research using MTS according to the study topic, there were 8 papers related to skin improvement and skin diseases, 7 papers related to hair growth and hair diseases, 3 papers about stability, and 1 paper about peripheral facial paralysis.

3. Among the animal research, 1 of them studied the dermal proliferative effect and stability of MTS, 2 of them studied the stability of MTS, 3 of them studied the hair growth effect of MTS, and 1 of them studied the optimal length and cycle of MTS for the hair growth effect.

4. Clinical trials mainly studied the skin improvement effect.

5. Of the case reports, there were 4 patients with acne scars; 2 patients with AGA; and 1 patient each with acne, acne, aging skin, peripheral facial paralysis, and pediatric complex type hair loss. In the case studies, in addition to MTS, various treatments such as subcision, herbal medicine, acupuncture, pharmacopuncture, etc. were used concurrently.

6. MTS treatment showed a hair growth effect and skin improvement effect in most studies. Moreover, significant effects were confirmed in the treatment of acne, acne scars, and hair loss. No serious side effects were observed, and most of the erythema and needle marks disappeared the next day of the treatment. Safety assessment confirmed that MTS was safe for use.

\section{CONFLICTS OF INTEREST}

The authors declare no conflict of interest.

\section{FUNDING}

This work was supported by the National Research Foundation of Korea (NRF) grant funded by the Korea government (MSIT) (No. 2020R1A2C1013956).

\section{ORCID}

Hea-Sun Chun, https://orcid.org/0000-0002-1002-9312

Ho-Seub Song, https://orcid.org/0000-0001-5306-8795

\section{REFERENCES}

1. Prausnitz MR. Microneedles for transdermal drug delivery. Adv Drug Deliv Rev. 2004;56(5):581-7.

2. Al-Qallaf B, Das DB. Optimizing microneedle arrays for trans- 
dermal drug delivery: extension to non-square distribution of microneedles. J Drug Target. 2009;17(2):108-22.

3. Aust MC, Fernandes D, Kolokythas P, Kaplan HM, Vogt PM. Percutaneous collagen induction therapy: an alternative treatment for scars, wrinkles, and skin laxity. Plast Reconstr Surg. 2008;121(4):1421-9.

4. Seong EJ, Jo EH, Park MC. A clinical report on the rejuvenate treatment with derma stamp. J Korean Orient Med Ophthalmol Otolaryngol Dermatol. 2010;23(3):225-35.

5. Kim JH, Park HY, Jung M, Choi EH. Dermal proliferative effect and safety of automicroneedle therapy system (AMTS). Korean J Dermatol. 2010;48(11):955-65.

6. Park KY, Lee JW, Kim DH, Lim YY, Kim BJ, Kim MN. The stability of the disk-type microneedle in Skh-hairless-1 (Albino) mice. Korean J Asthma Allergy Clin Immunol. 2010;30(3):2227.

7. Park KY, Jang WS, Lim YY, Ahn JH, Lee SJ, Kim CW, et al. Safety evaluation of stamp type digital microneedle devices in hairless mice. Ann Dermatol. 2013;25(1):46-53.

8. Lee CH, Lee JY, Shin HJ, Ha KT, Seo HS, Jeong HS. Hair-growth promoting effect of microneedle roller therapy. Korean J Orient Physiol Pathol. 2014;28(1):16-21.

9. Ju BH, Yu SA, Kang KH, Lee SY. Effects of ganoderma lucidum extract ethanol extract and microneedle therapy system on hair growth in an alopecia model of C57BL/6N mice. J Pediatr Korean Med. 2014;28(2):72-87.

10. Kang NR, Yoon HJ, Ko WS. Effects of microneedle therapy system(MTS) and Hwangryeonhaedoktang pharmacopuncture solution on hair growth in an alopecia model of C57BL/6N mouse. J Korean Med Ophthalmol Otolaryngol Dermatol. 2016; 29(1):47-64.

11. Kim YS, Jeong KH, Kim JE, Woo YJ, Kim BJ, Kang H. Repeated microneedle stimulation induces enhanced hair growth in a murine model. Ann Dermatol. 2016;28(5):586-92.

12. Lee HJ, Lee EG, Kang S, Sung JH, Chung HM, Kim DH. Efficacy of microneedling plus human stem cell conditioned medium for skin rejuvenation: a randomized, controlled, blinded split-face study. Ann Dermatol. 2014;26(5):584-91.

13. Koo JS. A study on the skin improvement effect of ginseng extract with microneedle therapy system. Kor J Herbol. 2021;36(4): $31-9$.
14. Seong EJ, Jo EH, Park MC. A clinical report on the acne treatment with AMTS. J Korean Orient Med Ophthalmol Otolaryngol Dermatol. 2010;23(3):236-46.

15. Lee KS, Kim YR, Choi HS. Case study of treating acne scar using scar regeneration acupuncture therapy and micro-needle therapy. J Korean Acupunct Moxib Med Soc. 2012;29(4):81-91.

16. Lee UI, Kwon YJ, Kim HH, Yoo JH, Kim KW, Kang JW, et al. Clinical observation of effect on severity of symptoms and safety of auto-microneedle therapy in patients with peripheral facial paralysis. J Korean Acupunct Moxib Med Soc. 2012;29(4):35-42.

17. Ju BH, Choi YK, Yu SA, Lee SY. A case report on child with different types of alopecia. J Pediatr Korean Med. 2013;27(3):53-64.

18. Heo JE, Yun JM, Shin SH. Case study of treating acne scars using reconstruction of skin scars and auto microneedle therapy system. J Korean Med Ophthalmol Otolaryngol Dermatol. 2013; 26(4):127-34.

19. Kim HY, Hong SH, Park IH, Heo ES, Noh HM, Shin SH. Clinical study of twenty-seven case of treating acne scar using microneedle therapy and subcision combined with Beevenom pharmacopuncture therapy. J Korean Med Ophthalmol Otolaryngol Dermatol. 2015;28(3):101-13.

20. Lee HK, Cho SY, Kim KN, Choi YH, Lee JH, Kim JH. Comparative study on treatment of acne scar. J Korean Med Ophthalmol Otolaryngol Dermatol. 2016;29(1):93-102.

21. Ro BI, Son HO, Chun SW, Shin HC. Therapeutic effects of growth factor cocktail treatment in patients with androgenetic alopecia according to the depth of microneedle. Korean J Dermatol. 2016;54(3):184-9.

22. Ro BI, Lee SY, Kim JB, Shin HC. Therapeutic effects of growth factor cocktail including fibroblast growth factor 9 in patients with pattern hair loss. Korean J Dermatol. 2017;55(8):504-10.

23. Kim BJ, Lee YY, Hong HG, Lee DH, Heo CH, Kim JY, et al. A study on drug delivery using MTS(Microneedling Therapy System). Korean Dermatol Assoc Autumn Meet. 2006;58(2):116.

24. Moon HS, Kim SE, Ko DS, Lee YY. Comparison of the effect of generating collagen by microneedling and IPL on the skin of white mice. Korean Dermatol Assoc Autumn Meet. 2006;58(2):120.

25. Higo N. [Recent trend of transdermal drug delivery system development]. Yakugaku Zasshi. 2007;127(4):655-62. Japanese. 